\title{
Optimization of flue gas convective heat transfer with condensation in a rectangular channel
}

\author{
SONG WeiMing, MENG JiAn \& LI ZhiXin* \\ Key Laboratory for Thermal Science and Power Engineering of Ministry of Education, Department of Engineering Mechanics, Tsinghua Univer- \\ sity, Beijing 100084, China
}

Received August 26, 2010; accepted October 21, 2010

\begin{abstract}
Conservation equations of sensible entarnsy and latent entransy are established for flue gas convective heat transfer with condensation in a rectangular channel and the entransy dissipation expression is deduced. The field synergy equation is obtained on the basis of the extremum entransy dissipation principle for flue gas convective heat transfer with condensation. The optimal velocity field is numerically obtained by solving the field synergy equation. The results show that the optimal velocity field has multiple longitudinal vortices, which improve the synergy not only between the veloctiy and temperature fields but also between the velocity and vapor concentration fields. Therefore, the convective heat and mass transfers are significantly enhanced. Flow with multiple longitudinal vortices close to the optimal velocity field can be generated by discrete double-inclined ribs set in the rectangular channel. The numerical results show that the total heat transfer rate in the discrete double-inclined rib channel increases by $29.02 \%$ and the condensing heat transfer rate increases by $27.46 \%$ for $R e=600$ compared with the plain channel.
\end{abstract}

condensation, entransy, entransy dissipation, field synergy, longitudinal vortex

Citation: $\quad$ Song W M, Meng J A, Li Z X. Optimization of flue gas convective heat transfer with condensation in a rectangular channel. Chinese Sci Bull, 2011, 56: 263-268, doi: 10.1007/s11434-010-4270-5

In condensation-type water heaters and boilers using natural gas, the mass fraction of the vapor in the flue gas is about $7 \%-25 \%$. Tubular heat exchangers with fins are often used to recover latent heat of the vapor, resulting in higher thermal efficiency. In the heat exchangers, the vapor condenses on the fin surface if the local fin surface temperature is lower than the dew point temperature. Therefore, there is both sensible heat transfer via the temperature difference and latent heat transfer via the vapor concentration difference during condensation, as for convective heat transfer with vapor condensation. There has been much experimental research [1-8] on convective heat transfer with condensation. However, there has been little theoretical study with the purpose of determining the heat transfer mechanism.

Bejan [9] analyzed convective heat transfer considering entropy generation based on irreversible thermodynamics

*Corresponding author (email: lizhx @ tsinghua.edu.cn) and optimized convective heat transfer according to the minimum entropy generation (MEG) principle. However, the heat transfer rate should be of much greater interest during heat transfer. Guo et al. $[10,11]$ introduced a physical quantity describing the heat transfer ability of an object, entransy, which is defined as half the product of the internal energy and temperature of the object. The extremum entransy dissipation (EED) principle was also established for heat transfer optimization. The optimization results [12-17] showed that the EED principle was more suitable than the MEG principle for heat transfer optimization. On the basis of the EED principle, Meng et al. [18] deduced the field synergy equation for laminar convective heat transfer using the variation principle for given input viscous dissipation. Their numerical results showed that the optimal velocity field was a multiple longitudinal vortex flow, and a new enhanced tube was developed to generate multiple longitudinal vortices. Song et al. [19] obtained the optimal velocity 
field for laminar convective heat transfer in a rectangular channel. The heat transfer was significantly enhanced by longitudinal vortex flow. Chen et al. [20] defined moisture transfer resistance and optimized liquid desiccant dehumidification. Recently, Liu et al. [21] studied the physical quantity synergy in a laminar flow field and its application in heat transfer enhancement. Chen et al. [22] extended the field synergy principle to the fluid flow and optimized the drag reduction.

In the present study, the flow channel of the finned tubular heat exchanger is simplified into a narrow rectangular channel and the flue gas is considered as a mixture of vapor and non-condensable gas. The flue gas flow in the channel is laminar with Reynolds numbers less than 1000. Firstly, entransy and entransy dissipation are defined for the convective heat transfer with vapor condensation. Secondly, the field synergy equation for laminar convective heat transfer with condensation is deduced using the EED principle. Thirdly, the optimal velocity field is numerically obtained for given input viscous dissipation. Finally, the enhancement of the convective heat transfer with condensation is validated setting discrete double-inclined ribs on the channel surfaces, which generate longitudinal vortices close to the optimal velocity field.

\section{Entransy and entransy dissipation}

Chen et al. [23] introduced the conservation equations of sensible entransy and latent entransy for the evaporative cooling system. Moisture entransy and moisture entransy dissipation were defined. For convective heat transfer with condensation, similarly, we establish entransy conservation equations and deduce the entransy dissipation expression.

Figure 1 shows the flue gas convective heat transfer with condensation in a rectangular channel. The vapor at high temperature condenses on the channel wall at low temperature.

The flue gas convective heat transfer must satisfy the governing equations.

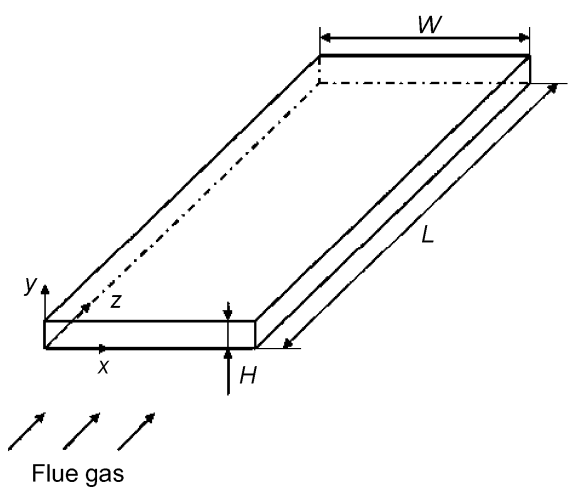

Figure 1 Flue gas convective heat transfer with condensation in a rectangular channel.
Energy equation:

$$
\rho U \cdot \nabla\left(c_{p} T\right)=\nabla \cdot(\lambda \nabla T)
$$

where $\rho, c_{p}$ and $\lambda$ are the density, specific heat and thermal conductivity of the flue gas, respectively. $U$ is the velocity vector and $T$ is the temperature. Eq. (1) is also the sensible heat conservation equation of the flue gas.

Species equation:

$$
\rho U \cdot \nabla \omega=\nabla \cdot(\rho D \nabla \omega) .
$$

where $\omega$ is the mass fraction of the vapor and $D$ is the mass diffusion coefficient of the vapor in the flue gas.

The vaporization latent heat of water, $\gamma$, is assumed to be constant. Multiplying both sides of eq. (2) by $\gamma$ gives the latent heat conservation equation:

$$
\rho U \cdot \nabla(\gamma \omega)=\nabla \cdot[\rho D \nabla(\gamma \omega)] .
$$

Multiplying both sides of eq. (1) by $T$ gives the conservation equation of the sensible heat entransy:

$$
\rho U \cdot \nabla g_{\mathrm{s}}=\nabla \cdot(\lambda T \nabla T)-\lambda(\nabla T)^{2},
$$

where $g_{s}=c_{p} T^{2} / 2$ is the sensible heat entransy per unit mass of the flue gas. The sensible heat entransy describes the sensible heat transfer ability of the flue gas. $g_{\mathrm{s}, \mathrm{dis}}=\lambda(\nabla T)^{2}$ is the sensible heat entransy dissipation. Eq. (4) indicates that the sensible heat entransy rate via convection equals the sensible heat entransy rate via conduction minus the sensible heat entransy dissipation rate.

Likewise, multiplying both sides of eq. (3) by $T$ gives

$$
T \rho U \cdot \nabla(\gamma \omega)=\nabla \cdot[\rho D T \nabla(\gamma \omega)]-\rho D \nabla T \cdot \nabla(\gamma \omega) .
$$

Assuming a linear relationship between the mass fraction of the vapor and its corresponding dew point temperature, $T_{\mathrm{dp}}=a \omega+b$, eq. (5) can be written as a conservation equation of the latent heat entransy:

$$
\begin{aligned}
\rho U \cdot \nabla g_{1}= & \nabla \cdot[\rho D T \nabla(\gamma \omega)] \\
& -\rho D \nabla T \cdot \nabla(\gamma \omega)-\left(T-T_{\mathrm{dp}}\right) \nabla \cdot[\rho D \nabla(\gamma \omega)],
\end{aligned}
$$

where $g_{1}=\gamma \omega T_{\mathrm{dp}} / 2$ is the latent heat entransy per unit mass of the flue gas. The latent heat entransy describes the latent heat transfer ability of the flue gas. The left side of eq. (6) is the latent heat entransy in the flue gas flow. The first item on the right side of eq. (6) represents the latent heat entransy diffusion induced by vapor diffusion. The second item is the latent heat entransy dissipation associated with not only the vapor concentration field but also the temperature field. The last item is the latent heat entransy diffusion due to the difference between the flue gas temperature and dew point temperature.

Combining eqs. (4) and (6) yields the entransy conserva- 
tion equation for the flue gas convective heat transfer:

$$
\begin{aligned}
\rho U \cdot \nabla g= & \nabla \cdot[\lambda T \nabla T+\rho D T \nabla(\gamma \omega)]-g_{\text {dis }}, \\
g_{\text {dis }}= & \lambda(\nabla T)^{2}+\rho D \nabla T \cdot \nabla(\gamma \omega) \\
& +\left(T-T_{\mathrm{dp}}\right) \nabla \cdot[\rho D \nabla(\gamma \omega)],
\end{aligned}
$$

where $g=c_{p} T^{2}+\gamma \omega T_{\mathrm{dp}} / 2$, as a measure of the total heat transfer ability of the flue gas, is the entransy per unit mass, and $g_{\text {dis }}$ is the total entransy dissipation.

\section{Field synergy equation}

Similar to the optimization for single-phase convective heat transfer $[18,19]$, the optimization objective for the convective heat transfer with condensation is to determine the optimal velocity field that leads to the extremum entransy dissipation for given viscous dissipation. Meanwhile, the flow and heat transfer are also governed by the continuity, energy and species equations. This is a typical functional extremum problem. Employing the calculus of variations, a Lagrange function is constructed with the given constraints:

$$
\Pi=\iiint_{\Omega}\left\{\begin{array}{l}
g_{\mathrm{dis}}+C_{0} \phi+A\left[\nabla \cdot(\lambda \nabla T)-\rho c_{p} U \cdot \nabla T\right] \\
+B[\nabla \cdot(\rho D \nabla \omega)-\rho U \cdot \nabla \omega]+C \nabla \cdot(\rho U)
\end{array}\right\} \mathrm{d} V,
$$

where $\phi$ is the viscous dissipation rate per unit volume and $C_{0}, A, B$ and $C$ are Lagrange multipliers. Owing to the different types of constraints, $C_{0}$ remains constant for given viscous dissipation, while $A, B$ and $C$ are functions of $U, T$, $\omega$ and local position.

The variation in $\prod$ with respect to $T$ gives the governing equation of $A$ :

$$
-\rho c_{p} U \cdot \nabla A=\nabla \cdot(\lambda \nabla A)-2 \nabla \cdot(\lambda \nabla T) .
$$

The boundary conditions are $A=0$ for given boundary temperatures and $\partial A / \partial n=2 \partial T / \partial n+\rho D \gamma / \lambda \partial \omega / \partial n$ for given boundary heat flux.

The variation in $\Pi$ with respect to $\omega$ gives the governing equation of $B$ :

$$
-\rho U \cdot \nabla B=\nabla \cdot(\rho D \nabla B)-2 a \nabla \cdot(\rho D \gamma \nabla \omega) .
$$

The boundary conditions are $B=-\chi\left(T-T_{\mathrm{dp}}\right)$ for given boundary species and $\partial B / \partial n=a \gamma \partial \omega / \partial n$ for a given boundary species gradient.

The variation in $\Pi$ with respect to $U$ is

$$
\mu \nabla^{2} U+\frac{\rho c_{p}}{2 C_{0}} A \nabla T+\frac{\rho}{2 C_{0}} B \nabla \omega+\frac{\rho}{2 C_{0}} \nabla C=0 .
$$

Comparing eq. (12) with the momentum equation

$$
\rho U \cdot \nabla U=-\nabla P+\nabla \cdot(\mu \nabla U)
$$

gives

$$
C=-2 C_{0} P / \rho,
$$

where $C_{0}$ is related to the viscous dissipation. Eq. (12) can be rewritten as

$$
\rho U \cdot \nabla U=-\nabla P+\mu \nabla^{2} U+F,
$$

where

$$
F=\rho U \cdot \nabla U+\frac{\rho c_{p}}{2 C_{0}} A \nabla T+\frac{\rho}{2 C_{0}} B \nabla \omega .
$$

Eq. (15) is Euler's equation governing the fluid velocity and temperature fields under the EED principle during convective heat transfer with condensation. Essentially, it is the momentum equation with a special additional volume force $F$, which improves the synergy not only between the velocity and temperature fields but also between the velocity and vapor concentration fields. Therefore, the heat transfer is enhanced for given viscous dissipation.

For given boundary conditions, the continuity, energy and species equations and eqs. (10), (11) and (15), including the six variables $U, T, \omega, P, A$ and $B$, can be solved together to provide the optimal velocity field, which gives the extremum entransy dissipation for given viscous dissipation.

\section{Numerical model}

Figure 1 shows the flue gas convective heat transfer in a rectangular channel with length $L=80 \mathrm{~mm}$, width $W=40$ $\mathrm{mm}$ and height $H=5 \mathrm{~mm}$. The convective heat transfer with condensation can be numerically optimized using the field synergy equation given above. There are four assumptions in the numerical model.

(1) Neglecting the presence of droplets and liquid film implies, in principle, that the condensate is promptly removed from the channel wall.

(2) When the local channel wall temperature is lower than the corresponding dew point temperature, there is condensation and the saturation value of the mass fraction is utilized as a Dirichlet boundary condition for the species conservation equation. In addition, the ideal gas relationship is used to compute the mass fraction of the vapor corresponding to the saturation pressure at the absolute wall temperature.

$$
\omega_{\mathrm{w}}=P_{\mathrm{s}} /\left(\rho R_{\mathrm{g}, \mathrm{v}} T_{\mathrm{w}}\right)
$$

where $R_{\mathrm{g}, \mathrm{v}}$ is the gas constant of the vapor, and the approximate relationship used to evaluate the saturation pressure is

$$
P_{\mathrm{s}}=610.78 \exp \left[17.2694\left(T_{\mathrm{w}}-273.15\right) /\left(T_{\mathrm{w}}-34.58\right)\right] .
$$

(3) The transverse velocity component induced by the 
condensation is disregarded. Therefore, the no-slip condition is used for the wall surface.

(4) When there is condensation, both the amounts of flue gas and vapor are reduced and latent heat is released. In the numerical model, the mass, species and energy sources are added to the boundary cells where the vapor condenses. Mass and species sources are $S_{\mathrm{m}}=-m_{\mathrm{v}} A_{\text {face }} / V_{\text {cell }}$ and the energy source is $S_{\mathrm{e}}=m_{\mathrm{v}} \gamma A_{\text {face }} / V_{\text {cell }} \cdot m_{\mathrm{v}}=-\rho D(\partial \omega / \partial n)_{\mathrm{w}}$ is the condensing rate of the vapor. $A_{\text {face }}$ is the interface area of the boundary cell and $V_{\text {cell }}$ is the boundary cell volume.

In the simulation, the flue gas temperature, velocity and mass fraction of the vapor are given at the inlet. $T_{\mathrm{in}}=440 \mathrm{~K}$, $R e=400, \omega=10 \%$. The channel wall temperature is constant; $T_{\mathrm{w}}=300 \mathrm{~K}$. Fully developed distributions of variables $A$ and $B$ are assumed at the outlet extended $300 \mathrm{~mm}$ downstream with zero gauge pressure.

The commercial computational fluid dynamics code FLUENT6.0 was used to solve the governing equations. The SIMPLEC algorithm was used for the velocity-pressure coupling. The QUICK scheme was employed for the discretization of the convection and diffusion terms. A user-defined function is used for varying properties of the flue gas with different temperature, adding the additional volume force in the momentum equation, governing equations and corresponding boundary conditions of variables $A$ and $B$, species boundary conditions of the vapor on the channel wall and adding sources to the boundary cells.

\section{Optimization results}

The convective heat transfer with condensation in the rectangular channel is numerically optimized. The total viscous dissipation, total heat transfer rate, condensing heat transfer rate and condensing proportion before and after optimization with $C_{0}=-5.0 \times 10^{6} \mathrm{~K}$ are listed in Table 1 .

Table 1 shows that, compared with the case before optimization, the total viscous dissipation of the flue gas flow in the channel after optimization only increases by $3.35 \%$, while the total heat transfer rate increases by $31.67 \%$ and the condensing heat transfer rate and condensing proportion of the vapor increase by $29.26 \%$. Therefore, both the sensible and latent heat transfers are significantly enhanced after optimization.

The velocity vectors, distributions of the temperature and mass fraction of the vapor at the cross section $z=40 \mathrm{~mm}$ in the channel are shown in Figure 2. It is seen that the optimal flow pattern has multiple longitudinal vortices, which sig- nificantly affect the distributions of the temperature and vapor concentration.

Compared with the case before optimization, the gradients of the temperature and vapor concentration increase in the region where the flow washes upon the wall. The included angles between the velocity vector and the temperature gradient decrease, as do the included angles between the velocity vector and the vapor concentration gradient. The synergy not only between the velocity and temperature fields but also between the velocity and vapor concentration fields is improved. Therefore, the convective heat and mass transfer are enhanced. On the contrary, in the region where the flow is away from the wall, the gradients of the temperature and vapor concentration decrease. The included angles between the velocity vector and temperature gradient increase, as do the included angles between the velocity vector and vapor concentration gradient. The synergy not only between the velocity and temperature fields but also between the velocity and vapor concentration fields is reduced, resulting in less convective heat and mass transfer. For the whole channel, the flow with multiple longitudinal vortices significantly improves the synergy not only between the velocity and temperature fields but also between the velocity and vapor concentration fields, leading to enhanced convective heat and mass transfer. Therefore, the flue gas convective heat transfer with condensation can be significantly enhanced by longitudinal vortices with little increment in viscous dissipation.

\section{Discrete double-inclined rib channel}

The numerical analysis shows that flow with multiple longitudinal vortices can enhance convective heat transfer with

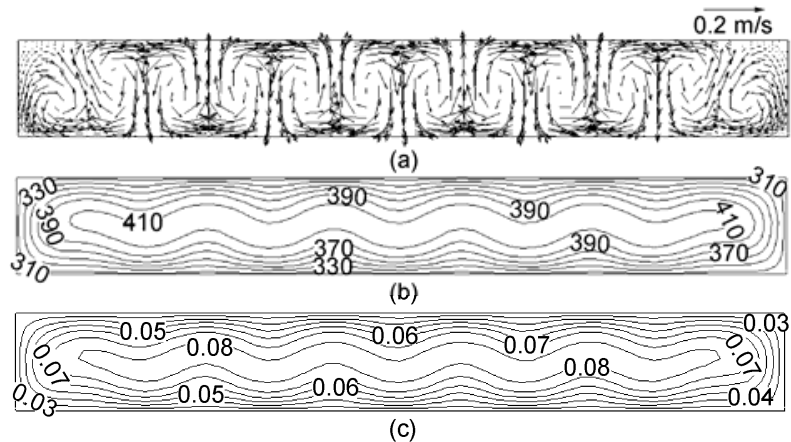

Figure 2 Optimization results for the flue gas convective heat transfer with condensation. (a) Velocity vectors; (b) temperature distribution (unit: $\mathrm{K})$; (c) mass fraction of the vapor.

Table 1 Optimization results for flue gas convective heat transfer with condensation

\begin{tabular}{|c|c|c|c|c|}
\hline & Viscous dissipation (W) & Total heat transfer rate $(\mathrm{W})$ & Condensing heat transfer rate $(\mathrm{W})$ & Condensing proportion \\
\hline Before optimization & $2.57 \times 10^{-4}$ & 30.95 & 12.55 & $23.70 \%$ \\
\hline After optimization & $2.66 \times 10^{-4}$ & 40.75 & 16.22 & $30.63 \%$ \\
\hline
\end{tabular}


condensation. Therefore, certain engineering techniques, such as the application of internal ribs, can be adopted to produce a desired velocity field close to the optimal one so as to enhance the heat transfer with condensation. Longitudinal vortex flow can be generated in tubes [18,24,25] and rectangular channels $[19,26]$ using discrete double-inclined ribs, resulting in enhanced heat transfer. Figure 3 shows the structure of the discrete double-inclined rib channel, with a rib length $r=7 \mathrm{~mm}$, rib width $g=3 \mathrm{~mm}$, rib pitch $p=15$ $\mathrm{mm}$ and attack angle $\alpha=45^{\circ}$. Here, the flue gas convective heat transfer with condensation is numerically studied.

Because of the complex flow inside the discrete double inclined-rib channel, turbulence may originate even at a low Reynolds number, especially near the rib region. In the case of heat transfer with a low Reynolds number less than 1000, Meng et al. [27] showed that the numerical results predicted by the RNG $k-\varepsilon$ model were more accurate than those predicted by the laminar model. Thus, the RNG $k-\varepsilon$ model was utilized in the simulation. The velocity vectors, distributions of the temperature and mass fraction of the vapor at the cross section $z=35 \mathrm{~mm}$ in the discrete double-inclined rib channel are shown in Figure 4. It is seen that longitudinal vortices close to the optimal velocity field are generated by the discrete double-inclined ribs. Therefore, the synergy between the velocity and temperature fields, as well as the synergy between the velocity and vapor concentration fields, is improved.

Compared with the plain channel, the simulation results show that the total heat transfer rate in the discrete doubleinclined rib channel increases by $29.02 \%$, the condensing heat transfer rate and the condensing proportion of the vapor increase by $27.46 \%$, while the friction increases by $60.69 \%$. Therefore, the optimal velocity field controlled by the field synergy equation can be used to guide the design and optimization of the convective heat transfer with condensation.

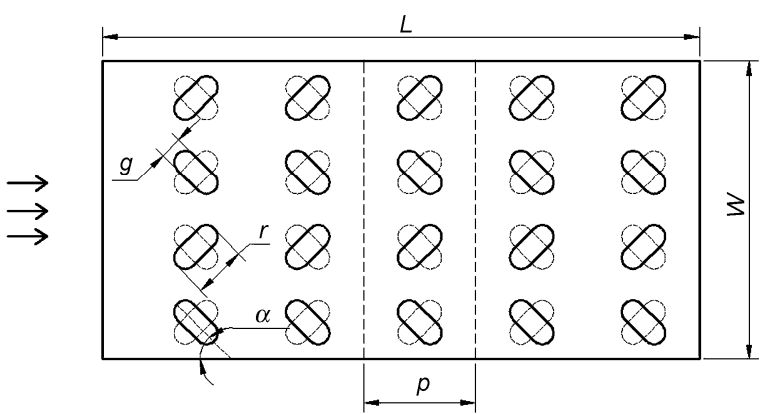

(a)

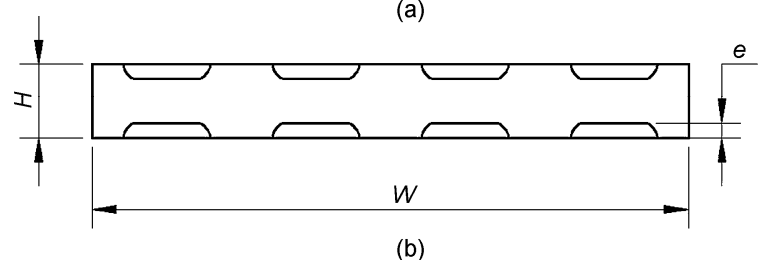

Figure 3 The discrete double-inclined rib channel. (a) Top view; (b) front view.

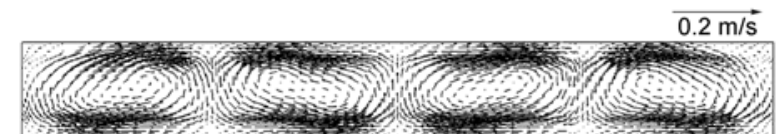

(a)

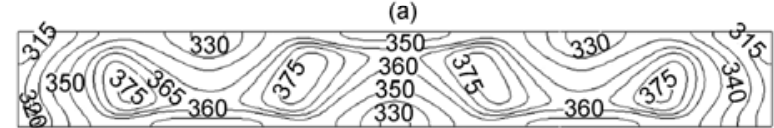

(b)

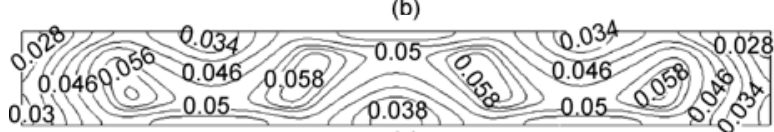

(c)

Figure 4 Velocity vectors, temperature distribution and mass fraction of the vapor at $z=35 \mathrm{~mm}$. (a) Velocity vectors; (b) temperature distribution (unit: K); (c) mass fraction of the vapor.

\section{Conclusions}

Entransy and entransy dissipation are defined for convective heat transfer with vapor condensation. The field synergy equation for the convective heat transfer with condensation is deduced on the basis of the EED principle.

Numerical results show that the optimal flow pattern has multiple longitudinal vortices, which significantly improve the synergy not only between the velocity and temperature fields but also between the velocity and vapor concentration fields. The flue gas convective heat transfer with condensation can be significantly enhanced by longitudinal vortices with little increment in viscous dissipation.

Flow with multiple longitudinal vortices close to the optimal velocity field can be generated by discrete double-inclined ribs arranged in the rectangular channel. The numerical results show that the total heat transfer rate in the rectangular channel with discrete double inclined ribs increases by $29.02 \%$ and the condensing heat transfer rate increases by $27.46 \%$ for $R e=600$ compared with the plain channel.

This work was supported by the National Basic Research Program of China (2007CB206901).

1 Idem S A, Jacobi A M, Goldschmidt V W. Heat transfer characterization of a finned-tube heat exchanger (with and without condensation). ASME J Heat Transfer, 1990, 112: 64-70

2 Kawaguchi K, Okui K, Shimoura T, et al. Heat transfer and pressure loss characteristics of a heat exchanger for recovering latent heat. Heat Transfer-Asian Res, 2001, 36: 230-247

3 Jia L, Peng X F, Sun J D, et al. An experimental study on vapor condensation of wet flue gas in a plastic heat exchanger. Heat TransferAsian Res, 2001, 30: 571-580

4 Cao Y B, Ai X Y, Guo Q, et al. Investigation on forced convective heat transfer with steam condensation of flue by experiment (in Chinese). J Eng Thermophys Chin, 2000, 21: 729-733

5 Cui Y Z, Qian S X. Study on condensing heat transfer of plate-fintube heat exchanger for nature gas flue (in Chinese). J Shandong Institute Arch Eng, 2000, 15: 41-45

6 Wang L. Study of heat exchanger in condensing gas-fired water heater (in Chinese). Master Dissertation. Shanghai: Tongji Univer- 
sity, 2006

7 Qi W. Study of heat transfer for wet mixture gas flowing over a finned tube (in Chinese). Master Dissertation. Beijing: Beijing Jiaotong University, 2007

8 Wu Y Y. Study on effective utilization of natural gas and heat and mass transfer (in Chinese). Master Dissertation. Beijing: Beijing University of Civil Engineering and Architecture, 2007

9 Bejan A. Advanced Engineering Thermodynamics. New York: Wiley, 1997

10 Guo Z Y, Zhu H Y, Liang X G. Entransy-A physical quantity describing heat transfer ability. Int J Heat Mass Transfer, 2007, 50: 2545-2556

11 Guo Z Y, Liang X G, Zhu H Y. Entransy-A physical quantity describing heat transfer ability (in Chinese). Prog Nat Sci, 2006, 16: 1288-1296

12 Wu J, Cheng X G, Meng J A, et al. Potential capacity dissipation extremum and entropy generation minimazation in laminar convective heat transfer (in Chinese). J Eng Thermophys Chin, 2006, 27: 100-102

13 Chen Q, Wu J, Ren J X. Thermodanamic optimization and heat transfer optimization for convective heat transfer (in Chinese). J Eng Thermophys Chin, 2008, 29: 271-274

14 Liu X B, Meng J A, Guo Z Y. Entropy generation extremum and entransy dissipation extremum for heat exchanger optimization. Chinese Sci Bull, 2009, 54: 943-947

15 Chen Q, Wang M R, Pan N, et al. Optimization principles for convective heat transfer. Energy, 2009, 34: 1199-1206

16 Liu X B, Guo Z Y. A novel method for heat exchanger analysis (in Chinese). Acta Phys Sin, 2009, 58: 4766-4771

17 Chen L, Chen Q, Li Z, et al. Optimization for a heat exchanger couple based on the minimum thermal resistance principle. Int $\mathrm{J}$ Heat
Mass Transfer, 2009, 52: 4778-4784

18 Meng J A, Liang X G, Li Z X. Field synergy optimization and enhanced heat transfer by multi-longitudinal vortices flow in tube. Int $\mathbf{J}$ Heat Mass Transfer, 2005, 48: 3331-3337

19 Song W M, Meng J A, Li Z X. Optimal velocity field for laminar convective heat transfer in rectangular channel (in Chinese). In: Proceedings of Heat and Mass Transfer by China Engineering Thermophysics Society, Tsingtao, 2009

20 Chen L, Chen Q, Li Z, et al. Moisture transfer resistance method for liquid desiccant dehumidification analysis and optimization. Chinese Sci Bull, 2010, 55: 1445-1453

21 Liu W, Liu Z C, Guo Z Y. Physical quantity synergy in laminar flow field of convective heat transfer and analysis of heat transfer enhancement. Chinese Sci Bull, 2009, 54: 3579-3586

22 Chen Q, Ren J X, Guo Z Y. Fluid flow field synergy principle and its application to drag reduction. Chinese Sci Bull, 2008, 53: 1768-1772

23 Chen Q, Yang K D, Wang M R, et al. A new approach to analysis and optimization of evaporative cooling system I: Theory. Energy, 2010, 35: 2448-2454

24 Li X W, Yan H, Meng J A, et al. Visualization of longitudinal vortex flow in an enhanced heat transfer tube. Exp Therm Fluid Sci, 2005, 31: 601-608

25 Li X W, Meng J A, Guo Z Y. Turbulent flow and heat transfer in discrete double inclined ribs tube. Int J Heat Mass Transfer, 2009, 52: 962-970

26 Song W M, Meng J A, Li Z X. Numerical study of air-side performance of a finned flat tube heat exchanger with crossed discrete double inclined ribs. Appl Therm Eng, 2010, 30: 1807-1814

27 Meng J A, Liang X G, Chen Z J, et al. Experimental study on convection heat transfer in alternating elliptical axis tubes. Exp Therm Fluid Sci, 2005, 29: 457-465

Open Access This article is distributed under the terms of the Creative Commons Attribution License which permits any use, distribution, and reproduction in any medium, provided the original author(s) and source are credited. 